\title{
IMPROVING MOTOR IMAGERY EEG SIGNALS CLASSIFICATION ACCURACY WITH CSP BY AVAILABLE MACHINE LEARNING APPROACH
}

\author{
Umme Farhana $^{1 *}$ and Mst. Jannatul Ferdous ${ }^{2}$ \\ ${ }^{1,2}$ Department of Computer Science \& Engineering, Jatiya Kabi Kazi Nazrul Islam University, Mymensing- \\ 2224, Bangladesh
}

Received: 17 April $2021 \quad$ Accepted: 23 June 2021

\begin{abstract}
In brain computer interface (BCI) systems, the electroencephalography (EEG) signals give a pathway to a motor disabled person to communicate outside using the brain signal and a computer. EEG signals of different motor imagery (MI) movements can be differentiated using an effective classification technique to aid a motor disabled patient. The purpose of this paper is to classify two different types of MI movement tasks, movement of the left hand and movement of the right foot EEG signals accurately. For this purpose we have used a publicly available dataset. Since the feature extraction for classification is an important task, so we have used popular common spatial pattern (CSP) method for spatial feature extraction. Two different machine learning classifiers named support vector machine (SVM) and K-nearest neighbor (KNN) have been used to verify the proposed method. We got the highest average results $95.55 \%, 98.73 \%$ and $92.38 \%$ in case of SVM and $93.5 \%, 98.73 \%$ and $90.15 \%$ in case of KNN for classification accuracy, sensitivity, and specificity, respectively when a Butterworth band-pass filter passed through [10-30] Hz. On the other hand accuracy came to 89.4\% in [10-30] Hz when applying CSP for feature extraction and fisher linear discriminant analysis (FLDA) for classification on this dataset earlier.
\end{abstract}

Keywords: Brain Computer Interface; Common Spatial Pattern; Electroencephalography; Fisher Linear Discriminant Analysis; K-Nearest Neighbor; Motor Imagery; Support Vector Machine.

\section{INTRODUCTION}

BCI system is simply a communicating system that allows a person to establish a link between the brain and an exterior device, i.e. computer (Ramesh et al., 2014). According to Isa et al. (2019), the progress of the BCI system depends on the effective role of signals in classification. This system is mostly feasible for motor disabled patient such as for a paralysed patient. Because they are unable to perform the actual bodily movement, but they are capable to imagine a type of bodily movement and as a result MI based EEG signals are generated. So we need to classify different MI bodily movement EEG signals to lead to the BCI system. Some MI movement tasks such as left hand, right hand, and foot are mostly used in a BCI system (Pfurtscheller and Neuper, 2001). Several clinical processes, such as electroencephalography (EEG), functional magnetic resonance imaging (fMRI), magnetoencephalography (MEG), positron emission tomography (PET) have been applied to detect the brain activities during MI mental task according to Sadiq et al. (2019). That paper also mentioned that electroencephalography is the most broadly used because of its easy usage and cheap setup. When the mental brain activity of movement is identifying, then this activity changes into a command associated to this activity in BCI that control an outer device such as a computer, a robot, a keyboard, or a wheelchair (Teplan, 2002). After all, the BCI system qualifies a motor disabled patient to lead his/her life without help of others.

Brain waves are classified according to certain frequency bands; delta: 0.5-4 Hz, theta: 4-8 Hz, alpha: 8-12 Hz, beta: $12-30 \mathrm{~Hz}$, gamma: $>30 \mathrm{~Hz}$ (Palaniappan, 2010). Afrakhteh and Mosavi (2020) mentioned that the expected frequency band for MI classification is $8-30 \mathrm{~Hz}$ (alpha and beta bands). Belhadj et al. (2015) showed the classification accuracy with different frequency bands and but good results came in 10-30 Hz. We have considered two different bands of 8-30 Hz and 10-30 Hz at two times and good results came in $10-30 \mathrm{~Hz}$. It is observed that when a motor imaginary movement happened, both the brain rhythms alpha and beta over the contralateral motor cortex are used but beta band is mostly used. So we took some from the alpha band and the full frequency from the beta band. Many papers have worked on the classification of left hand and right hand MI movement but have not found much work on left hand and right foot MI movement. Alomari et al. (2013), Hossain et al. (2015), Rashid et al. (2016), Huong et al. (2018), Gaur et al. (2019) and more others have worked to classify left hand and right hand MI movement based EEG data. That's why we worked with left hand and right foot.

In this study, we used publicly available benchmark dataset IVb from BCI competition III. Belhadj et al. (2015) found accuracy highly reached at $89.4 \%$ after applying CSP for feature extraction and FLDA for classification on 
this dataset earlier where each channel of the left hand and right foot data had been passed through the $20^{t^{\text {th }}}$ order Butterworth band-pass filter. Lavanya et al. (2016) used Parks-Mcclellan FIR filter, Marrugo et al. (2018) a FIR equiripple filter and IIR elliptic filter and Saha et al. (2019) IIR bandpass filter in pre-processing step. The feature extraction term describing EEG signals by some pertinent values called 'features' (Bashashati et al., 2007). The feature extraction is essential to reduce noise and extract effective features in signal processing (Rabcan et al., 2020). Li P. et al. (2013) mentioned that the CSP method finds the spatial filters that can enhance the variance of one class and decrease the variance of other class at the same time and got the same number of features for two classes. So the features of two different classes can be easily differentiated and this may increase the accuracy of classification. Clemente et al., (2019) and Ma et al., (2016) found CSP log-variance features. We have considered CSP variance features. To improve the classification accuracy, we present a CSP method with a spatial filtering function to correctly extract spatial features and classification has done by SVM and also KNN. Saha et al. (2019) also worked with CSP and they considered four trials data but we've found good results by considering three trials that are time-saving. The SVM is a popular machine learning classifier that classifies at least two tasks, based on the building of a hyper plane that divides all the features per class (Li S. et al., 2013). It is easy to implement both SVM and KNN classifiers with MATLAB.

\section{DATASET}

We used openly available dataset IVb from BCI competition III delivered by Fraunhofer FIRST, the Intelligent Data Analysis Group and Campus Benjamin Franklin of Charité-University Medicine Berlin, the Department of Neurology, Neurophysics Group (Dornhege et al., 2004).

\subsection{Dataset Description}

The EEG data are taken from one healthy person. The dataset holds data from the 7 preliminary sessions with no feedback where the first 3 sessions are presented with labels. Visual signs were given for 3.5 seconds, according to two motor imagery tasks that are accomplished by the subject: (F) for the right foot, (L) for the left hand. The remaining consecutive sessions (4 to 7) are given without any stimulus information class label. During these sessions, the tasks left hand, right foot and relaxation have been ordered by acoustic stimuli for periods between 1.5 and 8 seconds. The signal recording was done with BrainAmp amplifiers and a 128-channel Ag/AgCl electrode cap from ECI. 118 EEG channels were measured at positions of international 10/20-system. The mental state left hand is used to position the cursor at -1 , and the right foot is used to position the cursor near +1 (Dornhege et al., 2004).

\subsection{Dataset Preparation}

The real data length is $210259 \times 118$ for both classes. The EEG segments from both classes have been considered that are only responsible for MI tasks. Though the original dataset was recorded with $1000 \mathrm{~Hz}$, but we considered the down-sampled version of the dataset at $100 \mathrm{~Hz}$ sampling frequency. We took $3.5 \mathrm{sec}$ from each EEG trial segment for both classes that produced 350 samples (3.5x100). Therefore, finally we got a data matrix of 350x 118 for each EEG segment for each class. Based on the markers provided with the dataset, the data was split into right foot (RF) and left hand (LH) movement classes using corresponding class labels; 1 for RF and -1 for LH. Using the class labels, in a total of 210 trials, for the right foot, 105 trials and 105 trials found for the left hand. From 118 channels 30 channels $F C 5, F C 3, F C 1, F C 2, F C 4, F C 6, C F C 5, C F C 3, C F C 1, C F C 2, C F C 4, C F C 6, C 5, C 3$, $C 1, C 2, C 4, C 6, C C P 5, C C P 3, C C P 1, C C P 2, C C P 4, C C P 6, C P 5, C P 3, C P 1, C P 2, C P 4$ and $C P 6$ were chosen manually for both classes, which are accountable to control the execution of MI tasks.

\section{METHODS AND PERFORMANCE METRICS}

Figure 1 illustrates the block diagram of our proposed work. This diagram consists of some different phases to find out the class ID that belongs to either left hand or the right foot.

\subsection{Pre-processing}

Pre-processing is the primary step. Each channel of right foot data has been passed through the $4^{\text {th }}$ order Butterworth band-pass filter [10 - 30] Hz. Similarly, left hand data also passed through the Butterworth bandpass filter. Also, the purpose of band-pass filtering is to remove some artefacts arising from eye blinks and other unexpected bodily movements. 


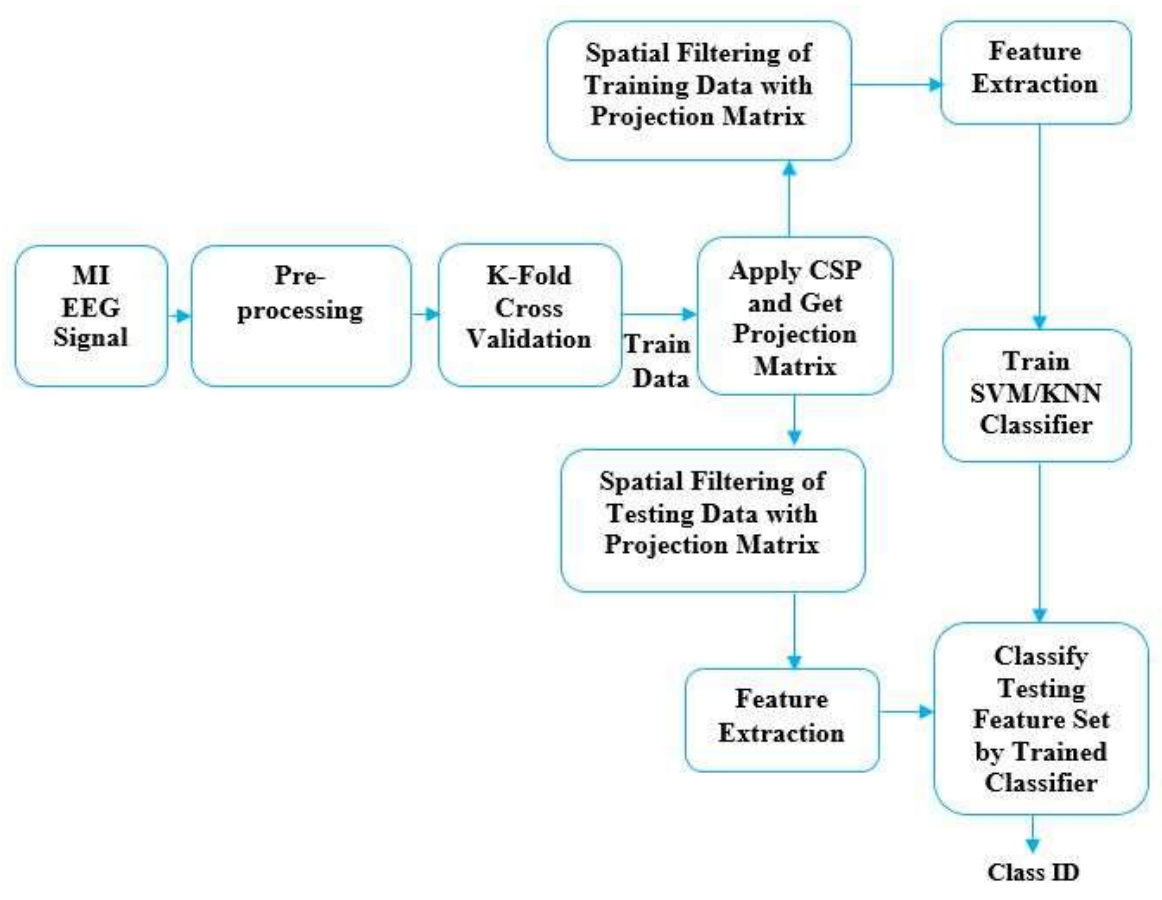

Figure 1: Block diagram of proposed methodology.

Right foot MI movement of $F C 5, F C 3, F C 1, F C 2, F C 4$ and $F C 6$ channel's noisy signals and Butterworth filtered signals are shown in Fig. 2(a) and Fig. 2(b), respectively.

Left hand MI movement of FC5, FC3, FC1, FC2, FC4 and FC6 channel's noisy signals and Butterworth filtered signals are shown in Fig. 3(a) and Fig. 3(b), respectively.
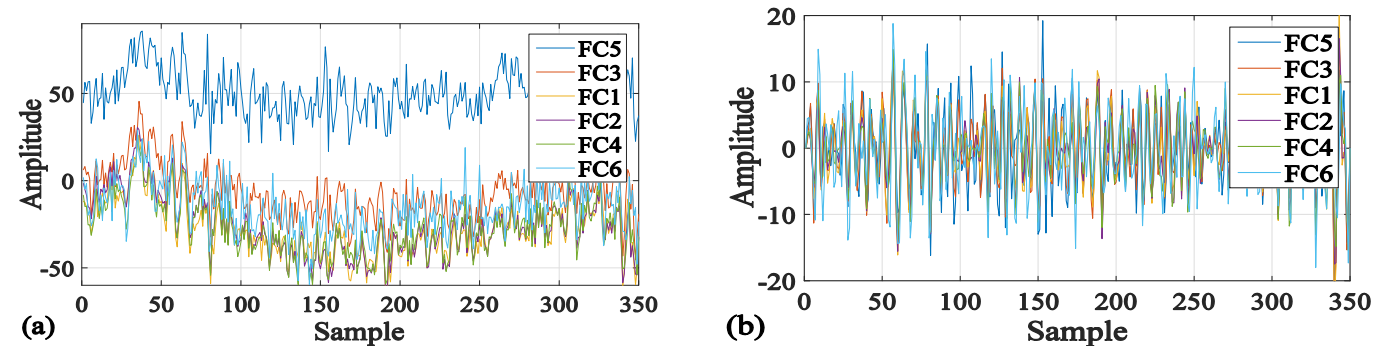

Figure 2: Right foot signals of channels $F C 5, F C 3, F C 1, F C 2, F C 4$ and FC6; (a) Non-filtered, (b) Butterworth filtered.
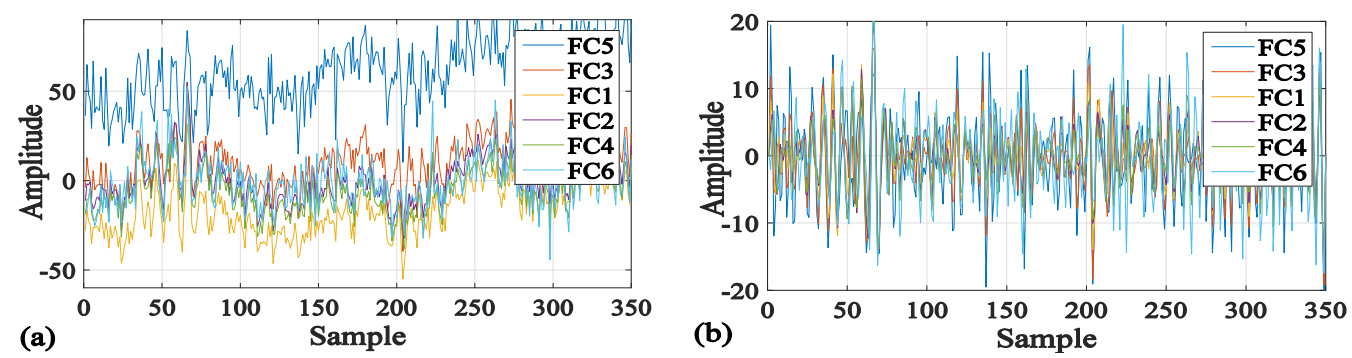

Figure 3: Left hand signals of channels FC5, FC3, FC1, FC2, FC4 and FC6; (a) Non-filtered, (b) Butterworth filtered. 


\subsection{Feature Extraction using Common Spatial Pattern}

Siuly et al. (2012) explained that feature extraction is the most important part because the classifier performance will be better when the features are extracted perfectly. The CSP is used to separate the two imaginary movements with the help of the number of applied channels (Marrugo et al., 2018). We divide the data of selected channels by 3 -fold cross validation for obtaining training and testing data.

At first, calculate normalized covariance matrices $C_{1}$ and $C_{2}$ for class 1 and class 2, respectively as follows:

$$
C_{1}=\frac{X_{1} X_{1}{ }^{T}}{\operatorname{trace}\left(X_{1} X_{1}{ }^{T}\right)} \text { and } C_{2}=\frac{X_{2} X_{2}{ }^{T}}{\operatorname{trace}\left(X_{2} X_{2}{ }^{T}\right)}
$$

Where $X_{1}$ and $X_{2}$ both denotes a training data trial of size [CxS], where $C$ is the number of channels and $S$ is the number of samples of class 1 and class 2 , respectively. $T$ for transpose matrices and trace function performs the diagonal sum of the elements.

Since we considered 3 trials of dataset, the spatial covariance matrices $\overline{C_{1}}$ and $\overline{C_{2}}$ for class 1 and class 2 , respectively have been calculated by averaging $C_{1}$ and $C_{2}$ over the trials as follows:

$$
\overline{C_{1}}=\frac{1}{3} \sum_{i=1}^{3} C_{1 i} \text { and } \overline{C_{2}}=\frac{1}{3} \sum_{i=1}^{3} C_{2 i}
$$

Compute the composite covariance matrix as follows-

$$
C_{\text {composite }}=\overline{C_{1}}+\overline{C_{2}}
$$

Perform generalized eigenvalue decomposition (GEVD) on it and found an eigenvector matrix $\left(E_{V}\right)$ and a diagonal eigenvalue matrix $\left(E_{\lambda}\right)$ and sort eigenvalue in descending order.

$$
\left[E_{V}, E_{\lambda}\right]=\operatorname{eig}\left(C_{\text {composite }}\right)
$$

The resulting $E_{V}$ and $E_{\lambda}$ matrices have been used to get the whitening transformation matrix ( $W$ ).

$$
W=\sqrt{E_{\lambda}^{-1}} E_{V}^{T}
$$

Whiten data $W 1$ and $W 2$ of class 1 and class 2 , respectively obtained by,

$$
W 1=W \overline{C_{1}} W^{T} \quad \text { and } \quad W 2=W \overline{C_{2}} W^{T}
$$

Where $W 1$ and $W 2$ are diagonal matrices and $W 1+W 2=I, I$ is the identity matrix and $W 1$ and $W 2$ share the common eigenvectors.

Now Generalized eigenvectors $\left(E_{V}\right)$ and eigenvalues $\left(E_{\lambda}\right)$ found using $W 1$ and $W 2$ and later sort the diagonal elements of $E_{\lambda}$.

$$
\left[E_{V}, E_{\lambda}\right]=\operatorname{eig}(W 1, W 2)
$$

If the first some eigenvalues are large that means there is a large difference in variances between two classes and it's easy to classify.

A projection matrix $(P)$ called the spatial filter coefficients, of size $[C x C]$, has been found as follows:

$$
P=E_{V}{ }^{T} W
$$

With the projection matrix $P$, the spatially filtered signals $X 1 \_C S P$ and $X 2 \_C S P$ of class 1 and class 2 , respectively can be obtained using a spatial filtering function.

$$
\begin{aligned}
& X 1 \_C S P=\operatorname{SpatFunc}(X 1, P, \text { Dim }) \\
& X 2 \_C S P=\operatorname{SpatFunc}(X 2, P, \text { Dim })
\end{aligned}
$$

Where $X 1$ and $X 2$ are EEG filtered training data of class 1 and class 2, respectively, Dim is the number of dimensions the filter should attempt to reduce the output vector space to.

Finally, the CSP variance based features are extracted from each trial. The variances of the first and last rows of $X 1 \_C S P$ and $X 2 \_C S P$ for class 1 and class 2 respectively are applicable features for classification. Then the features are joined together to make the feature vector of each trial. Feature vector of each trial of the train data is heap together to make the train feature matrix for training the SVM. The test feature matrix is obtained in the same process for testing the SVM classifier. We performed generalized eigenvalue decomposition (GEVD) on the composite covariance matrix and later whiten data for better performance. 
Fig. 4 and Fig. 5 show the outcome of CSP with spatial filtering function which applied to found two spatial filters that differentiate the right foot from the left hand MI data. After applying the CSP filters on C3 and C4, the output signals shown in Fig. 4(b) have larger variance in case of right foot motor imagery (red) while signals shown in Fig. 5(b) have smaller variance in case of left hand motor imagery (red). On the other hand, the signal shown in Fig. 4(b) have smaller variance in case of right foot motor imagery (green) while signals shown in Fig. 5(b) have larger variance in case of left hand motor imagery task (green).
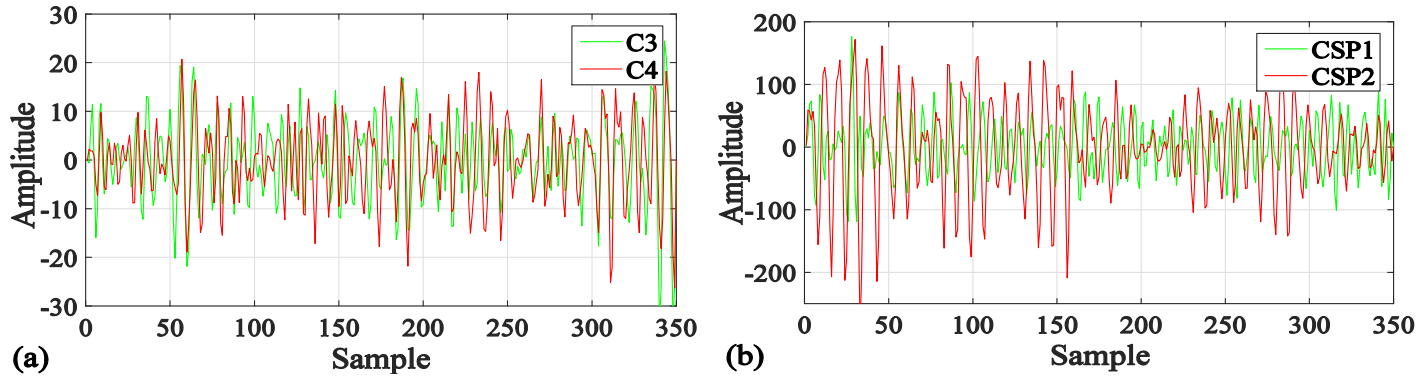

Figure 4: A single trial's C3 and C4 channels of the right foot training set; (a) before CSP filtering, (b) after CSP filtering, for $\mathrm{k}=1$ fold.
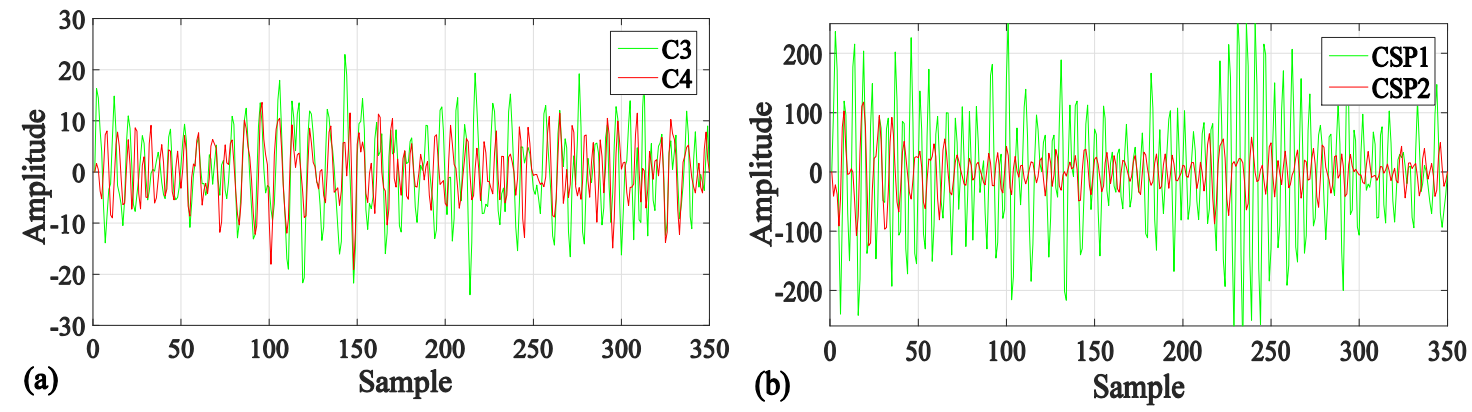

Figure 5: A single trial's C3 and C4 channels of the left hand training set; (a) before CSP filtering, (b) after CSP filtering, for $\mathrm{k}=1$ fold.

The distribution of training samples of class 1 (RF) and class 2 (LH) before and after CSP filtering are shown in Fig. 6(a) and 6(b), respectively. We see that both classes are deeply assembled before CSP filtering and but after executing the CSP, they are disparate at the same time.
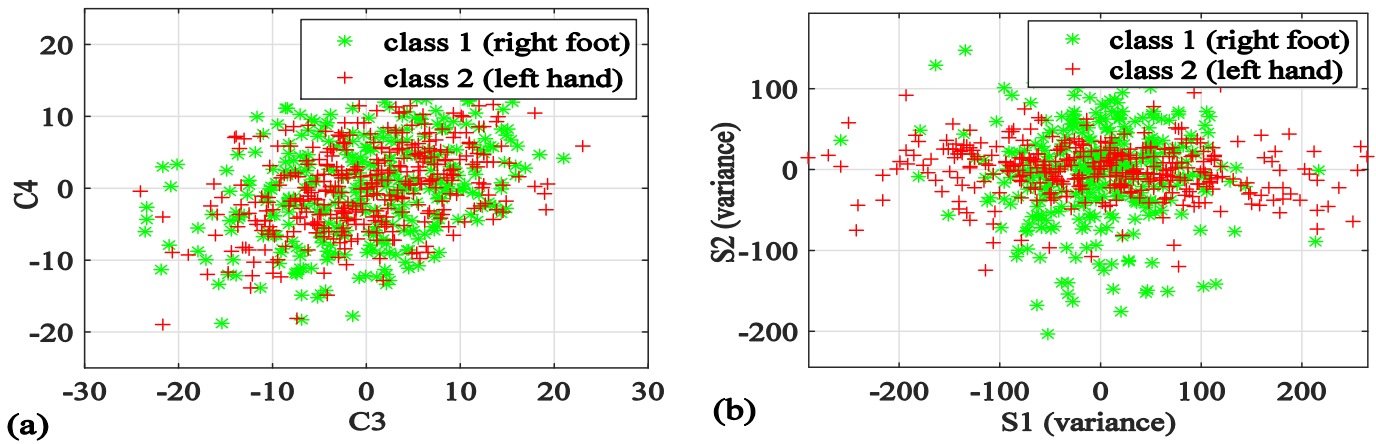

Figure 6: For two trials from the training set for $\mathrm{k}=1$ fold, class 1 (RF) and class 2 (LH); (a) before CSP filtering, (b) after CSP filtering.

In Fig. 6(b) the horizontal axis provides the largest variance for class 2 (red) and the smallest for class 1 (green) at the same time. On the other hand, the vertical axis provides the largest variance for class 1 (green) and the smallest for class 2 (red) at the same time. So after CSP filtering the variance of one class is maximized while minimizing the variance of the other class at the same time and vice versa. 

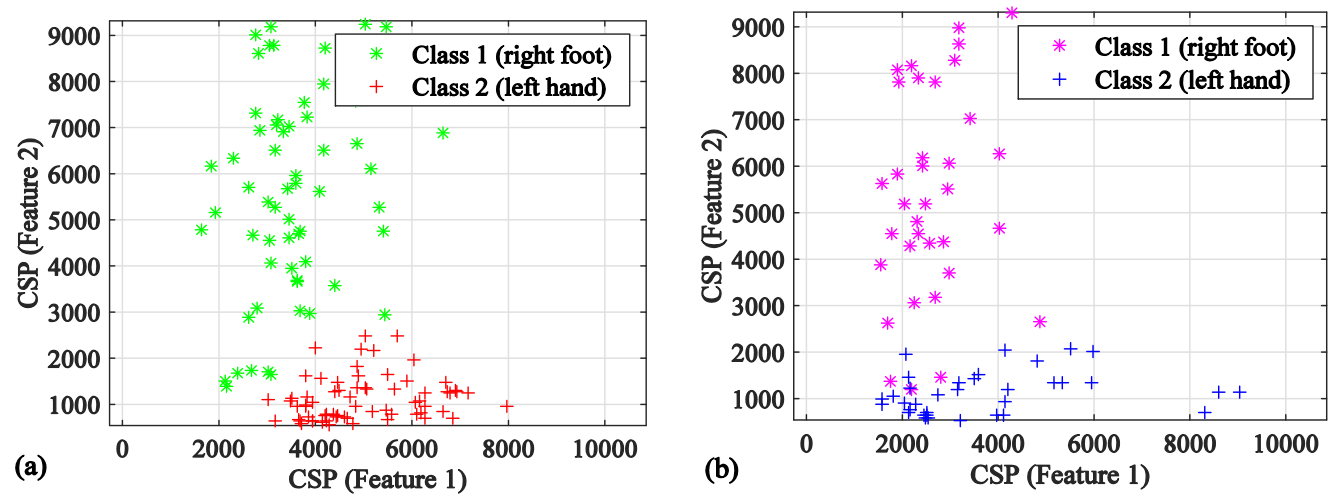

Figure 7: (a) CSP training features of the right foot and left hand, (b) CSP testing features of the right foot and left hand; respectively for the trial run $=1$ and $\mathrm{k}=1$ fold.

The CSP variance based training and testing features are shown in Fig. 7(a) and 7(b), respectively when the trial run $=1$ of the right foot and left hand for $\mathrm{k}=1$ fold. The 'green' and 'red' colours represent the training features of the right foot and left hand, respectively. The 'magenta' and 'blue' colours represent the testing features of the right foot and left hand, respectively.

\subsection{Classification using Support Vector Machine}

According to Lotte et al. (2007), a classification process simply determine a class of a set of features extracted from the signals. SVM classifier is a strong machine learning classifier that requires labelled data and widely used in MI based BCI systems either in binary (Youg et al., 2008), (Jurcak et al., 2007), or multi-class (Bose et al., 2016) problems.

In this work, we used two built-in SVM functions. Firstly, svmtrain() used for training the SVM model with CSP 'training' feature matrix and later, svmclassify() used for classifying each row of the CSP 'testing' feature matrix. Each row of 'testing' matrix represents an observation and each column represents a feature. SVM model has been evaluated with the 'testing' feature matrix. The svmclassify() function takes results from the svmtrain() that helps to classify feature vector ' $v$ ' using the Eq. 11.

$$
a=\sum_{j} w_{j} k\left(s_{j}, v\right)+b
$$

Where $s_{j}=$ support vectors, $w_{j}=$ weights, $b=$ bias and $k=$ kernel function. Selim et al. (2018) and Sadiq et al. (2019) used a radial basis function (RBF) kernel. Rayatnia et al. (2019) worked with linear, polynomial, RBF and sigmoid kernels and got a better feedback in case of linear kernel. We have considered a linear kernel function. Vector ' $v$ ' is classified as a member of class 1 when $a \geq 0$, otherwise it is classified as a member of class 2 . The SVM classifier trained with the training features of the right foot and left hand and these are separated with a hyper plane shown in Fig. 8 and evaluated with the help of testing features shown in Fig. 9; for a single trial of $\mathrm{k}=1$ fold.

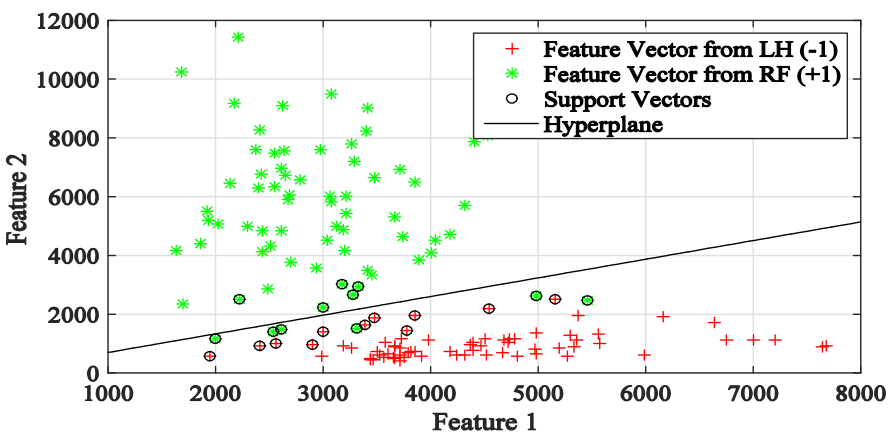

Figure 8: SVM separates the training features of the right foot (green) and left hand (red) with a hyper plane for the trial run=1 and $\mathrm{k}=1$ fold. 


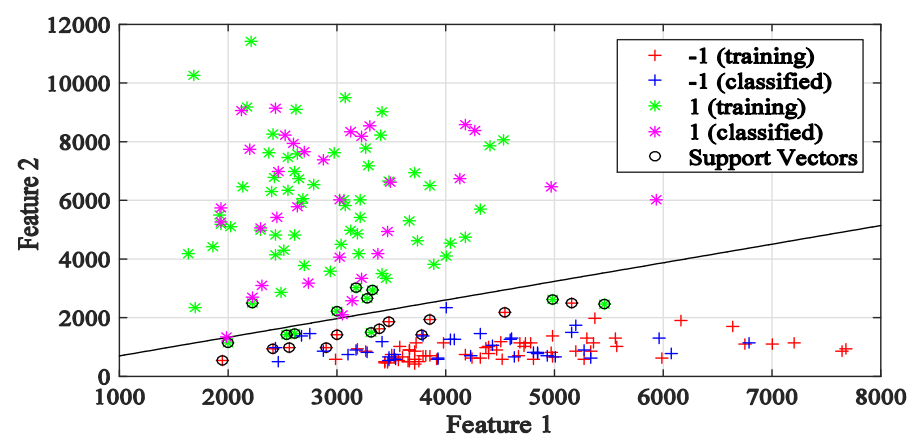

Figure 9: Trained SVM classified testing features of the right foot (magenta) and left hand (blue) for the trial run $=1$ and $\mathrm{k}=1$ fold.

\subsection{Classification using K- Nearest Neighbor}

KNN classifier based on searching of K-nearest neighbors in the feature space to classify new features. This classification algorithm needs training feature set and also need previously defined ' $\mathrm{K}$ ' value to find the class of new features. According to Isa et al., (2017), the new features will be classified by considering the maximum number of neighbor's class. If we consider $\mathrm{K}=7$, it means that the new feature classes depend on the nearest seven neighbors class labels within a particular distance. Let 4 neighbors under the class 1 and 3 neighbors under the class 2 among 7 neighbors. Then the new feature belongs to class 1 . In this work, ' $\mathrm{K}$ ' value ranges from 1 to 10. We performed KNN algorithm with the help of Euclidean distance metrics to find the distance between test vector and the nearest neighbour (Pan et al., 2004). The Euclidean distance metrics D $(a, b)$ between two points ' $a$ ' and ' $b$ ' in the feature space has been found using the Eq. 12. KNN classifier trained with the help of fitcknn() and verified by predict() function in MATLAB.

$$
D(a, b)=\sqrt{\sum_{i=1}^{k}\left(a_{i}-b_{i}\right)^{2}}
$$

\subsection{Performance Evaluation}

Some statistical parameters named classification accuracy, sensitivity or true positive rate, specificity or true negative rate, are used to assess the performance of the proposed method. The accuracy is the percentage of the test set which is accurately classified with the classifier. The sensitivity is the ratio of the positive set correctly defined. The specificity is the ratio of the negative set correctly identified (Ghayab et al., 2016). A confusion matrix is created for accuracy, sensitivity and specificity for each fold. Ubeyli et al. (2010) explained that the confusion matrix recognizes the common misclassifications of classification method. The accuracy, sensitivity and specificity are calculated for each fold using Eq. 13, 14 and 15, respectively.

$$
\begin{aligned}
& \text { Accuracy }=\left(\frac{T P+T N}{T P+T N+F P+F N}\right) \times 100 \\
& \text { Sensitivity }=\left(\frac{T P}{T P+F N}\right) \times 100 \\
& \text { Specificity }=\left(\frac{T N}{T N+F P}\right) \times 100
\end{aligned}
$$

Where TP means true positive that indicates the number of accurately calculated right foot MI tasks, while TN means true negative that represents the number of accurately calculated left hand MI tasks. FP means false positive and FN means false negative that represents the number of misclassified left hand and right foot MI tasks, respectively.

\section{RESULT ANALYSIS AND DISCUSSION}

In this work, all of the methods have been implemented in MATLAB R2014b. This paper shows all the figures and table data when the frequency range was 10-30 Hz. Nandi et al. (2021) mentioned that the classifier categorized the classes of one diagonal entry of confusion matrix accurately and other diagonal entries classified inaccurately for binary classification. Table 1 shows the confusion matrix for $k=1$ fold in case of SVM classifier. Here four values of the right foot are misclassified i.e. going to the left hand class. On the other hand, no values of the left hand are misclassified. The overall accuracy rate reaches at $94.28 \%$. Table 2 shows the confusion matrix for $\mathrm{k}=1$ fold and $\mathrm{K}=9$ nearest neighbors in case of $\mathrm{KNN}$ classifier and the overall accuracy rate reaches at $92.85 \%$. 
Table 1: Confusion matrix for $\mathrm{k}=1$ fold by SVM

\begin{tabular}{ccccc}
\hline & \multicolumn{2}{c}{$\begin{array}{c}\text { Considered } \\
\text { outcomes }\end{array}$} & $\begin{array}{c}\text { Classification } \\
\text { accuracy rate } \\
(\%)\end{array}$ \\
\hline Observed & RF & 31 & 4 & 88.57 \\
values & RF & LH & \\
& LH & 0 & 35 & 100 \\
Average & & & & 94.28 \\
\hline
\end{tabular}

Table 2: Confusion matrix for $\mathrm{k}=1$ fold by $\mathrm{KNN}$

\begin{tabular}{ccccc}
\hline & \multicolumn{2}{c}{$\begin{array}{c}\text { Considered } \\
\text { outcomes }\end{array}$} & $\begin{array}{c}\text { Classification } \\
\text { accuracy rate } \\
(\%)\end{array}$ \\
\hline Observed & RF & 31 & 4 & 88.57 \\
values & LH & 1 & 34 & 97.14 \\
Average & & & & 92.85 \\
\hline
\end{tabular}

Table 3: 3 - fold cross validation results for trial 1 by SVM

\begin{tabular}{cccc}
\hline Fold & $\begin{array}{c}\text { Accuracy } \\
(\%)\end{array}$ & $\begin{array}{c}\text { Sensitivity } \\
(\%)\end{array}$ & $\begin{array}{c}\text { Specificity } \\
(\%)\end{array}$ \\
\hline 1 & 97.14 & 97.14 & 97.14 \\
2 & 92.85 & 100 & 85.71 \\
3 & 97.14 & 100 & 94.28 \\
Avg. of 3 folds & 95.71 & 99.04 & 92.38 \\
\hline
\end{tabular}

Table 4: 3 - fold cross validation results for trial 2 by SVM

\begin{tabular}{cccc}
\hline Fold & $\begin{array}{c}\text { Accuracy } \\
(\%)\end{array}$ & $\begin{array}{c}\text { Sensitivity } \\
(\%)\end{array}$ & $\begin{array}{c}\text { Specificity } \\
(\%)\end{array}$ \\
\hline 1 & 97.14 & 94.28 & 100 \\
2 & 92.85 & 100 & 85.71 \\
3 & 95.71 & 100 & 91.42 \\
Avg. of 3 folds & 95.23 & 98.09 & 92.38 \\
\hline
\end{tabular}

Table 5: 3 - fold cross validation results for trial 3 by SVM

\begin{tabular}{cccc}
\hline Fold & $\begin{array}{c}\text { Accuracy } \\
(\%)\end{array}$ & $\begin{array}{c}\text { Sensitivity } \\
(\%)\end{array}$ & $\begin{array}{c}\text { Specificity } \\
(\%)\end{array}$ \\
\hline 1 & 94.28 & 100 & 88.57 \\
2 & 97.14 & 97.14 & 97.14 \\
3 & 95.71 & 100 & 91.42 \\
Avg. of 3 folds & 95.71 & 99.04 & 92.38 \\
\hline
\end{tabular}

The Tables 3, 4 and 5 show the 3-fold cross validation results of accuracy, sensitivity and specificity of dataset IVb for trial 1, 2 and 3, respectively after implementing SVM classification technique. Finally, Fig. 10 shows the 3 -fold cross validation results of accuracy, sensitivity and specificity for the overall 3 trials. 
Belhadj et al. (2015) considered only five channels and calculated only the classification accuracy for performance evaluation. Any method can't be properly verified with just one performance measurement criteria. They didn't follow the k-fold cross validation technique, but we did. Using 3-fold cross validation we easily performed training and validation. We considered thirty effective channels and calculated sensitivity and specificity along with classification accuracy. Table 6 shows 3 -fold cross validation results for the overall 3 trials when K-nearest neighbor value ranges from 1 to 10 in case of $\mathrm{KNN}$ classifier.

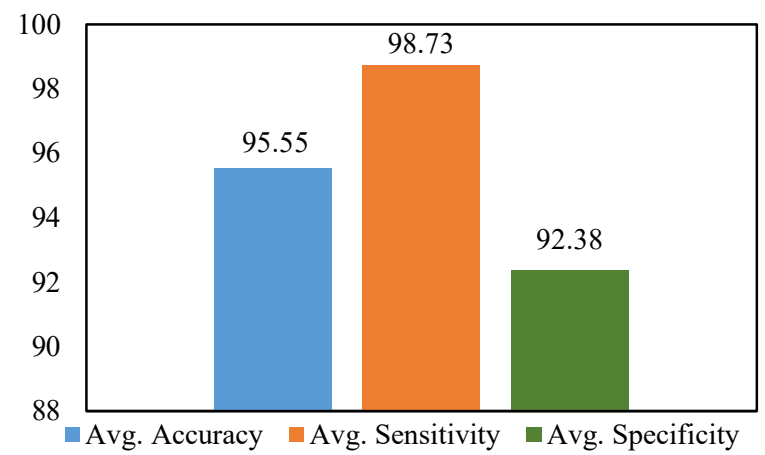

Figure 10: 3- fold cross validation results for the overall 3 trials by SVM.

Table 6: 3 - fold cross validation results for the overall 3 trials by KNN

\begin{tabular}{cccc}
\hline Value of K & $\begin{array}{c}\text { Avg. Accuracy } \\
(\%)\end{array}$ & $\begin{array}{c}\text { Avg. Sensitivity } \\
(\%)\end{array}$ & $\begin{array}{c}\text { Avg. Specificity } \\
(\%)\end{array}$ \\
\hline $\mathrm{K}=1$ & 89.68 & 89.20 & $\mathbf{9 0 . 1 5}$ \\
$\mathrm{K}=2$ & 90.79 & 95.87 & 85.71 \\
$\mathrm{~K}=3$ & 90.63 & 92.38 & 88.88 \\
$\mathrm{~K}=4$ & 91.58 & 96.2 & 86.98 \\
$\mathrm{~K}=5$ & 91 & 93.96 & 88.25 \\
$\mathrm{~K}=6$ & 92.22 & 97.14 & 87.3 \\
$\mathrm{~K}=7$ & 92.38 & 96.82 & 87.93 \\
$\mathrm{~K}=8$ & 93 & $\mathbf{9 8 . 7 3}$ & 87.3 \\
$\mathrm{~K}=9$ & $\mathbf{9 3 . 5}$ & 96.82 & $\mathbf{9 0 . 1 5}$ \\
$\mathrm{K}=10$ & 92.53 & 97.77 & 87.3 \\
\hline
\end{tabular}

Table 7: Comparative analysis of the proposed work with the existing work of dataset IVB

\begin{tabular}{ccccc}
\hline Methods & $\begin{array}{c}\text { Frequency } \\
\text { Band }\end{array}$ & $\begin{array}{c}\text { Accuracy } \\
(\%)\end{array}$ & $\begin{array}{c}\text { Sensitivity } \\
(\%)\end{array}$ & $\begin{array}{c}\text { Specificity } \\
(\%)\end{array}$ \\
\hline CSP + FLDA (Belhadj et al., 2015) & {$[7-30] \mathrm{Hz}$} & 85.3 & - & - \\
& {$[10-30] \mathrm{Hz}$} & 89.4 & - & - \\
CSP + KNN & {$[8-30] \mathrm{Hz}$} & 92.69 & 96.51 & 88.89 \\
& {$[10-30] \mathrm{Hz}$} & 93.5 & $\mathbf{9 8 . 7 3}$ & 90.15 \\
CSP + SVM (proposed) & {$[8-30] \mathrm{Hz}$} & 93.34 & 98.42 & 88.26 \\
& {$[10-30] \mathrm{Hz}$} & $\mathbf{9 5 . 5 5}$ & $\mathbf{9 8 . 7 3}$ & $\mathbf{9 2 . 3 8}$ \\
\hline
\end{tabular}

Table 7 shows that classification accuracy with our proposed methods (CSP+SVM) increased by $6.15 \%$ compared to the previous methods (CSP+FLDA) in [10-30] $\mathrm{Hz}$ that applied to the same dataset that we used. In case of KNN, accuracy increased by $4.1 \%$ and specificity increased by $2.23 \%$, i.e. SVM performed well than KNN classifier.

\section{CONCLUSIONS}

This paper has showed a feature extraction method to extract features and then performed classification on these features by two different machine learning classifiers. Filtering has been done by the $4^{\text {th }}$ order Butterworth bandpass filter successfully. The CSP method was applied on training data and got a projection matrix. Filtered signal 
and projection matrix passed through a spatial filtering function and got the required spatially filtered signal. The extracted training features from the spatially filtered signal are used to train the classifiers and evaluate with a new testing feature set successfully. SVM classifier is a desired selection for classifying the right foot and left hand movements since we gained the better average results $95.55 \%, 98.73 \%$ and $92.38 \%$ for classification accuracy, sensitivity and specificity, respectively for dataset IVb. This will be helpful in the medical field. The classifier performed well because of better feature extraction, and finally got better accuracy. The future work focuses on further research on EEG signal processing and its link with a different bodily movement.

\section{ACKNOWLEDGEMENTS}

The authors of this research work would like to thank ICT Division, the Ministry of Postal, Telecommunication and Information Technology of government of Bangladesh for financially supporting by their fellowship. Also grateful to the Dataset provider.

\section{REFERENCES}

Alomari M.H., Samaha A., and AlKamha K., 2013., Automated Classification of L/R Hand Movement EEG Signals using Advanced Feature Extraction and Machine Learning, International Journal of Advanced Computer Science and Applications, 4(6).

Afrakhteh S. and Mosavi M.R., 2020. “Applying an Efficient Evolutionary Algorithm for EEG Signal Feature Selection and Classification in Decision-Based Systems" in Energy Efficiency of Medical Devices and Healthcare Applications, Elsevier, 25-52, doi: https://doi.org/10.1016/B978-0-12-819045-6.00002-9.

Belhadj S. A., Benmoussad N., and Krachai M. D., 2015. CSP Features Extraction and FLDA Classification of EEG-Based Motor Imagery for Brain-Computer Interaction, 4th International Conference on Electrical Engineering (ICEE), IEEE publisher, 1-6.

Bashashati A., Fatourechi M., Ward R.K., and Birch G.E., 2007. A survey of signal processing algorithms in brain-computer interfaces based on electrical brain signals, Journal of Neural engineering, 4(2), R35-57.

Bose R., Khasnobish A., Bhaduri S., and Tibarewala D. N., 2016. Performance Analysis of Left and Right Lower Limb Movement Classification from EEG, 3rd International Conference on Signal Processing and Integrated Networks (SPIN), 174-179.

Clemente R.M., Olias J., Cruces S., and Zarzoso V., 2019. Unsupervised Common Spatial Patterns, IEEE Transactions On Neural Systems And Rehabilitation Engineering, 21(10), 2135-2144.

Dornhege G., Blankertz B., Curio G., and Muller K., 2004. Boosting bit rates in noninvasive eeg single-trial classifications by feature combination and multiclass paradigms, Biomedical Engineering, IEEE Transactions on, 51(6), 993-1002.

Gaur P., Pachori R.B., Wang H., and Prasad G., 2019. Automatic Subject Specific Intrinsic Mode Function Selection for Enhancing Two-Class EEG based Motor Imagery-Brain Computer Interface, IEEE Sensors Journal, 14(19), doi: 10.1109/JSEN.2019.2912790.

Ghayab H.R., Li Y., Abdulla S., Diykh M., Wan X., 2016. Classification of epileptic EEG signals based on simple random sampling and sequential feature selection, Brain Informatics, SpringerOpen, 3(2), 85-91, doi: https: //doi.org/10.1007/s40708-016-0039-1.

Hossain A.B.M.A., Rahman M.W., and Riheen M.A., 2015. Left and Right Hand Movements EEG Signals Classification Using Wavelet Transform and Probabilistic Neural Network, International Journal of Electrical and Computer Engineering, 5(1), 92-101.

Huong N.T.M., Linh H.Q., and Khai L.Q., 2018, Classification of Left/Right Hand Movement EEG Signals Using Event Related Potentials and Advanced Features, 6th International Conference on the Development of Biomedical Engineering in Vietnam, Springer, 63, 209-215.

Isa N. E., Amir A., Ilyas M.Z., and Razalli M. S., 2019. Motor imagery classification in Brain computer interface (BCI) based on EEG signal by using machine learning technique, Bulletin of Electrical Engineering and Informatics, 8(1), 270.

Isa N. E., Amir A., Ilyas M.Z., and Razalli M. S., 2017. The Performance Analysis of K-Nearest Neighbors (K$\mathrm{NN})$ Algorithm for Motor Imagery Classification Based on EEG Signal, MATEC Web of Conferences, 140, 01024.

Jurcak V., Tsuzuki D., and Dan I., 2007. 10/20, 10/10, and 10/5 systems revisited: their validity as relative headsurface-based positioning systems, Neuroimage, 34(4), 1600-1611.

Lavanya T.H., Jyothi K.S., 2016. EEG Based Classification of Hand Movements using BCI, International Journal of Computer Science and Network, 5(4), 687-691.

Li P., Xu P., Zhang R., Guo L. and Yao D., 2013. L1 Norm based common spatial patterns decomposition for scalp EEG BCI, Biomed. Eng. 12-77. 
Li S., Zhou W., Yuan Q., Geng S. and Cai D., 2013. Feature extraction and recognition of ictal EEG using EMD and SVM, Comput. Biol Med. 43(7), 807-816, doi: 10.1016/j.compbiomed.2013.04.002.

Lotte F., Congedo M., Lécuyer A., Lamarche F., and Arnaldi B., 2007. A review of classification algorithms for EEG-based brain computer interfaces, Journal of Neural Engineering, 4(2), R1-R13, doi: 10.1088/17412560/4/2/R01.

Ma Y., Ding X., She Q., Luo Z., Potter T., and Zhang Y., 2016. Classification of Motor Imagery EEG Signals with Support Vector Machines and Particle Swarm Optimization, Computational and Mathematical Methods Medicine, 16, 8, doi: https://doi.org/10.1155/2016/4941235.

Marrugo N., Amaya D., and Ramos O., 2018. Support Vector Machine To Classify Features Of Motion Imaginary EEG, ARPN Journal of Engineering and Applied Sciences, 13(6), 2179.

Nandi A., Xhafa F., Subirats L., and Fort S., 2021. Real-Time Emotion Classification Using EEG Data Stream in E-Learning Contexts, Sensors, 21(5), 1589, doi: https://doi.org/10.3390/s21051589.

Pfurtscheller G., and Neuper C., 2001. Motor imagery and direct brain-computer communication, In: Proceedings of the IEEE, 7, 1123-1134.

Palaniappan R., 2010. Biological Signal Analysis, A textbbok, Ramaswamy Palaniappan and Ventus Publishing ApS.

Pan F, Wang B, Hu X, Perrizo W, 2004. Comprehensive vertical sample-based KNN/LSVM classification for gene expression analysis, J Biomed Inform, 37(4), 240-248, doi: 10.1016/j.jbi.2004.07.003.

Rashid M. M. O., and Ahmad M., 2016. Classification of motor imagery hands movement using levenbergmarquardt algorithm based on statistical features of EEG signal, 3rd International Conference on Electrical Engineering and Information Communication Technology, 1-6, doi: 10.1109/CEEICT. 2016.7873081.

Ramesh S., Krishna M. G., and Nakirekanti M., 2014. Brain Computer Interface System for Mind Controlled Robot using Bluetooth, International Journal of Computer Applications, 104(15), 20-23.

Rabcan J., Levashenko V., Zaitseva E., Kvassay M., 2020. Review of Methods for EEG Signal Classification and Development of New Fuzzy Classification-Based Approach, IEEE Access, 8, 189722.

Rayatnia A., Khanbabaie R., 2019. Common Spatial Patterns Feature Extraction and Support Vector Machine Classification for Motor Imagery with the SecondBrain, International Journal of Engineering, 32(9), 1284-1289.

Sadiq U.T., Yu X., Yuan Z., Fan Z., Rehman A.U, Li G., and Xiao G., 2019. Motor Imagery EEG Signals Classification Based on Mode Amplitude and Frequency Components Using Empirical Wavelet Transform IEEE Access, 7, 127678-127692, doi: 10.1109/ACCESS.2019.2939623.

Saha P.K., Rahman M.A., and Mollah M.N., 2019, Frequency Domain Approach in CSP based Feature Extraction for EEG Signal Classification, International Conference on Electrical, Computer and Communication Engineering, IEEE Access, 1-6, doi: 10.1109/ECACE.2019.8679463.

Siuly, Li Y., and Wen P., 2012. Analysis and classification of EEG signals, University of Southern Queensland, Australia [thesis], 21-56.

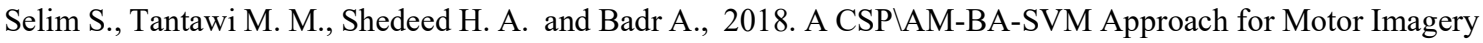
BCI System, IEEE Access, 6, 49192-49208, doi: 10.1109/ACCESS.2018.2868178.

Teplan M., 2002. Fundamentals of EEG Measurement, Measurement Science Review, 2(2).

Ubeyli E. D., 2010. Least Square Support Vector Machine Employing Model Based Methods coefficients for Analysis of EEG Signals, Expert System with Applications, 37(1), 233-239.

Youg X., Ward R. K., and Birch G. E., 2008. Sparse spatial filter optimization for eeg channel reduction in brain computer interface, IEEE International Conference on Acoustics, Speech and Signal Processing, 417 420 .

(C) 2021 the Authors. Journal of Engineering Science published by Faculty of Civil Engineering, Khulna University of Engineering \& Technology. This is an open access article under the terms of the Creative Commons AttributionNonCommercial-NoDerivatives License, which permits use and distribution in any medium, provided the original work is properly cited, the use is non-commercial and no Modifications or adaptations are made. 